\title{
NONEXISTENCE THEOREMS OF HIGHER ORDER IMIMERSIONS OF DOLD MANIFOLDS
}

\author{
TEIICHI KOBAYASHI ${ }^{1}$
}

\begin{abstract}
The purpose of this note is to improve partially the nonexistence theorems of J. J. Ucci and W.-L. Ting on immersions of Dold manifolds in Euclidean spaces.
\end{abstract}

1. Introduction. Since W. F. Pohl [7] and E. A. Feldman [1], [2] have investigated differential geometry of higher order, several authors have been studying higher order nonsingular immersions of manifolds (cf. [3], [5], [6], [8]-[10] etc.). W.-L. Ting [10] employs $\gamma$-operations and characteristic classes to prove a nonexistence theorem of higher order nonsingular immersions of Dold manifolds. The purpose of this paper is to prove other nonexistence theorems of higher order nonsingular immersions of Dold manifolds in Euclidean spaces using homological properties of stunted projective spaces. Our method can be used to obtain nonexistence theorems of higher order nonsingular immersions of lens spaces in Euclidean spaces [4].

2. Notations and theorems. Let $m$ and $n$ be integers and $p$ be a positive integer. Define integers $A$ and $B$ as follows:

$$
\begin{aligned}
& A=A(m, n, p)=\sum_{0<\text { odd } i<p}\left(\begin{array}{c}
n+p-i-1 \\
p-i
\end{array}\right)\left(\begin{array}{c}
m+n+i \\
i
\end{array}\right), \\
& B=B(m, n, p)=\sum_{0<\text { even } i<p}\left(\begin{array}{c}
n+p-i-1 \\
p-i
\end{array}\right)\left(\begin{array}{c}
m+n+i \\
i
\end{array}\right),
\end{aligned}
$$

where $\left(\begin{array}{c}m+n \\ n\end{array}\right)=(m+n) ! / m ! n !$. For example, $A(m, n, 1)=m+n+1, B(m, n, 1)$ $=n, A(m, n, 2)=n(m+n+1), B(m, n, 2)=\left(\begin{array}{c}n+1 \\ 2\end{array}\right)+\left(\begin{array}{c}m+n+2 \\ 2\end{array}\right)$. Notice that $A+$ $B=\left({ }_{p}^{m+2 n+p}\right)$.

For integers $l$ and $m$ with $l<m, \phi(m, l)$ denotes the number of integers $s$ such that $l<s<m$ and $s \equiv 0,1,2$, or $4 \bmod 8$. For the fixed $m$, we set

$$
\phi(l)=\phi(m, l-\varepsilon), \quad \psi(l)=\left[\left(m-l+\varepsilon^{\prime}\right) / 2\right],
$$

where $\varepsilon=0$ or 1 according as $l \equiv 0$ or $\not \equiv 0 \bmod 4$, and $\varepsilon^{\prime}=0$ or 1 according as $l \equiv 0$ or $\not \equiv 0 \bmod 2$. Here $[k]$ denotes the integral part of $k$. Let $\subseteq_{p}$ (or $\Phi_{p}$ ) denote existence (or nonexistence) of a $p$ th order nonsingular immersion. In case $p=1$, we write simply $\subseteq($ or $\nsubseteq)$ instead of $\subseteq_{1}$ (or $\Phi_{1}$ ).

Received by the editors December 17, 1979 and, in revised form, February 11, 1980.

AMS (MOS) subject classifications (1970). Primary 57D40.

Key words and phrases. Dold manifold, $p$ th order nonsingular immersion, $K$-theory, KO-theory, stunted projective spaces.

${ }^{1}$ The author wishes to express his thanks to the referee for his kind suggestions. 
Now we are ready to state our theorems. Let $P(m, n)$ be the $(m+2 n)$ dimensional Dold manifold of type $(m, n)$ (cf. [11]).

THEOREM 1. Let $N$ be an integer satisfying the following:

$\left(\begin{array}{c}m+2 n+p \\ p\end{array}\right) \leqslant N<\left(\begin{array}{c}m+2 n+p \\ p\end{array}\right)-1+m, \quad\left(\begin{array}{c}N-B+1 \\ A\end{array}\right) \neq 0 \bmod 2$.

Moreover, suppose that one of the next two conditions holds:

(i) $A \equiv 0 \bmod 2$, and $\neq 0 \bmod 2^{\psi(N-A-B+1)-1}$,

(ii) $A \equiv 0 \bmod 8$, and $\neq 0 \bmod 2^{\phi(N-A-B+1)-1}$.

Then $P(m, n) \Phi_{p} R^{N}$.

THEOREM 2. Let $N$ be an integer satisfying the following:

$\left(\begin{array}{c}m+2 n+p \\ p\end{array}\right)-1-m<N<\left(\begin{array}{c}m+2 n+p \\ p\end{array}\right)-2, \quad\left(\begin{array}{c}A-1 \\ N-B\end{array}\right) \neq 0 \bmod 2$.

Moreover, suppose that one of the next two conditions holds:

(i) $A \equiv 0 \bmod 2$, and $\neq 0 \bmod 2^{\psi(A+B-N-1)-1}$,

(ii) $A \equiv 0 \bmod 8$, and $\neq 0 \bmod 2^{\phi(A+B-N-1)-1}$.

Then $P(m, n) \Phi_{p} R^{N}$.

We give some examples to show that, in certain cases, our theorems contain sharper nonimmersion results than theorems obtained by means of Stiefel-Whitney classes and $\gamma$-operations.

Let $(m, n)=(30,1),(28,3)$ or $(24,7)$. Then the total Stiefel-Whitney class of $P(m, n)$ is equal to 1 (cf. [11, Corollary 3.4]), and this gives no information. By $\gamma$-operations [11, Theorem 2.12], we see $P(30,1) \nsubseteq R^{41}, P(28,3) \nsubseteq R^{43}, P(24,7) \nsubseteq$ $R^{45}$. On the other hand, we have, by Theorem $1, P(30,1) \nsubseteq R^{49}, P(28,3) \nsubseteq R^{51}$, $P(24,7) \nsubseteq R^{55}$.

The total Stiefel-Whitney class of the second order tangent bundle of $P(m, n)$ is equal to 1 (cf. [10, p. 199]), and this gives no information. By $\gamma$-operations [10, Corollary 4], we see $P(30,1) \varpi_{2} R^{560+k},-9<k<9 ; P(28,3) \varpi_{2} R^{629+k},-8<k$ $<8 ; P(24,7) \Phi_{2} R^{779+k},-6<k<6$. On the other hand, we have, by Theorems 1 and 2, $P(30,1) \Phi_{2} R^{560+k},-17<k<17 ; \quad P(28,3) \Phi_{2} R^{629+k}, \quad-17<k<17$; $P(24,7) \Phi_{2} R^{779+k},-11<k<11$.

3. Preliminaries. Let $\tau_{p}\left(M^{n}\right)$ be the $p$ th order tangent bundle of $M^{n}$. Then $\tau_{p}\left(M^{n}\right)$ is a smooth vector bundle of dimension $\nu(n, p)=\left(\begin{array}{c}n+p \\ p\end{array}\right)-1$. According to [2, I, Proposition 8.4] the following holds.

Proposition (3.1) (Feldman). Suppose $M^{n} \subseteq_{p} R^{N}$. (1) If $N>\nu(n, p)$, then there is an $(N-\nu(n, p))$-vector bundle $\alpha$ over $M^{n}$ such that $\tau_{p}\left(M^{n}\right) \oplus \alpha=N$, where $\oplus$ denotes the Whitney sum. (2) If $N<\nu(n, p)$, then there is $a(\nu(n, p)-N)$-vector bundle $\beta$ over $M^{n}$ such that $\tau_{p}\left(M^{n}\right)=\beta \oplus N$.

Let $R P^{m}$ denote the real projective $m$-space, $i: R P^{m}(=P(m, 0)) \rightarrow P(m, n)$ be the natural inclusion, and $\xi$ be the canonical line bundle over $R P^{m}$. W.-L. Ting obtains in [10, Theorem 4] the following formula from [11, Theorem 1.5] and [8, Lemma 2.2]. 
TheOREM (3.2) (TING). $i^{*} \tau_{p}(P(m, n))=A \xi+B-1 \in K O\left(R P^{m}\right)$.

In [3, Lemmas 4.1 and 4.2] we have the following two results.

LeMMA (3.3). Let $l$ be an integer with $0<l<m$. Suppose that $\left({ }^{l}+k\right) \neq 0 \bmod 2$. If the vector bundle $(l+k) \xi$ has $k$ independent cross-sections, then the stunted projective spaces $R P^{m} / R P^{l-1}$ and $R P^{m+k} / R P^{l-1+k}$ are $\bmod 2 S$-related. (Here two spaces $Y$ and $Z$ are said to be mod $2 S$-related if for some nonnegative integers $r$ and there is a map $S^{r} Y \rightarrow S^{t} Z$ which induces isomorphisms of all homology groups with $Z_{2}$-coefficients.)

LEMMA (3.4). Let $k$ be an integer such that $k \equiv 0 \bmod 8$. If $R P^{m} / R P^{l-1}$ and $R P^{m+k} / R P^{l-1+k}$ are $\bmod 2 S$-related, then $k \equiv 0 \bmod 2^{\phi(l)-1}$.

Using $K$-theory in place of $K O$-theory in the proof of (3.4), we obtain the following.

LEMMA (3.5). Let $k$ be even. If $R P^{m} / R P^{l-1}$ and $R P^{m+k} / R P^{l-1+k}$ are $\bmod 2$ $S$-related, then $k \equiv 0 \bmod 2^{\psi(l)-1}$.

\section{Proofs of theorems.}

Proof of TheOREM 1. Under the assumption of the theorem, suppose that $P(m, n) \subseteq_{p} R^{N}$. Since

$$
\nu(m+2 n, p)<N,
$$

by $(3.1)(1)$ there is an $(N-\nu(m+2 n, p))$-vector bundle $\alpha$ over $P(m, n)$ such that $\tau_{p}(P(m, n)) \oplus \alpha=N$. For the inclusion $i: R P^{m} \rightarrow P(m, n)$, we have $i^{*} \tau_{p}(P(m, n))$ $\bigoplus i^{*} \alpha=N$. Combining this equality with (3.2), it holds that $A \xi \oplus i^{*} \alpha=N-B$ +1 , since $N-B+1>m$. Multiplying both sides by $\xi$ and taking account of the fact that $\xi^{2}=1$, we have $A \oplus\left(i^{*} \alpha\right) \otimes \xi=(N-B+1) \xi$. It follows from the first part of the assumption that (3.3) is applicable to the vector bundle $(N-B+1) \dot{\xi}$. Therefore, if $A$ is even, then $A \equiv 0 \bmod 2^{\psi(N-A-B+1)-1}$ by (3.5), and if $A \equiv 0$ mod 8 , then $A \equiv 0 \bmod 2^{\phi(N-A-B+1)-1}$ by (3.4). This contradicts the second part of the assumption.

Proof of Theorem 2. Under the assumption of the theorem, suppose that $P(m, n) \subseteq_{p} R^{N}$. Since $\nu(m+2 n, p)>N$, by (3.1)(2) there is a $(\nu(m+2 n, p)-N)$ vector bundle $\beta$ over $P(m, n)$ such that $\tau_{p}(P(m, n))=\beta \oplus N$. Therefore we obtain $A \xi=i^{*} \beta \oplus(N-B+1)$ from (3.2), since $A>m$. Multiplying both sides by $\xi$, and then considering the equality $L \xi=L$ in $K O\left(R P^{m}\right)$ for some large integer $L$, we obtain $(L-N+B-1) \xi=\left(i^{*} \beta\right) \otimes \xi \oplus(L-A)$. But we have, for suitably chosen $L$,

$$
\left(\begin{array}{c}
L-N+B-1 \\
L-A
\end{array}\right) \equiv\left(\begin{array}{c}
A-1 \\
N-B
\end{array}\right) \bmod 2 .
$$

Thus we can apply (3.3) to the bundle $(L-N+B-1) \xi$, as desired. 


\section{REFERENCES}

1. E. A. Feldman, The geometry of immersions. I, II, Bull. Amer. Math. Soc. 69 (1963), 693-698; 70 (1964), 600-607. MR 27 \#1967; 29 \#625.

2. _ The geometry of immersions. I, II, Trans. Amer. Math. Soc. 120 (1965), 185-224; 125 (1966), 181 - 215. MR 32 \#3065; 34 \#818.

3. T. Kobayashi, On the odd order non-singular immersions of real projective spaces, J. Sci. Hiroshima Univ. Ser. A-1 33 (1969), 197-207. MR 41 \# 4561.

4. , Higher order nonsingular immersions of lens spaces mod 3, Mem. Fac. Sci. Kochi Univ. (to appear).

5. H. Ôike, Higher order tangent bundles of projective spaces and lens spaces, Tôhoku Math. J. 22 (1970), 200-209. MR 42 \#6857.

6. __ Inflection-free embeddings of Grassmann manifolds, Tôhoku Math. J. 28 (1976), 311-326. MR 54 \# 13938.

7. W. F. Pohl, Differential geometry of higher order, Topology 1 (1962), 169-211. MR 27 \#4242.

8. H. Suzuki, Bounds for dimensions of odd order nonsingular immersions of RP ${ }^{n}$, Trans. Amer. Math. Soc. 121 (1966), 269-275. MR 33 \# 733.

9. (1972), 161-170. MR 46 \#8239.

10. W.-L. Ting, On higher order nonsingular immersions of Dold manifolds, Proc. Amer. Math. Soc. 39 (1973), 195-200. MR 51 \# 14103.

11. J. J. Ucci, Immersions and embeddings of Dold manifolds, Topology 4 (1965), 283-293. MR 32 \#4703.

Department of Mathematics, Faculty of SCIEnCE, Koch University, Kochi, 780 Japan 